\title{
Hepatitis Lupoid or Lupus Hepatitis: A Challenging Diagnosis
}

\author{
Rosa Coelho ${ }^{1 *}$, Regina Gonçalves ${ }^{1}$, Joanne Lopes ${ }^{2}$, Guilherme Macedo ${ }^{1}$ \\ ${ }^{1}$ Gastrenterology Department, Centro Hospitalar São João, Oporto, Portugal \\ ${ }^{2}$ Pathology Department, Centro Hospitalar São João, Oporto, Portugal
}

Received: August 27, 2016; Accepted: September 20, 2016; Published: October 4, 2016

*Corresponding author: Rosa Coelho, Department of Gastroenterology, Centro Hospitalar São João, Oporto. Alameda Prof. Hernâni Monteiro 4200319 Porto, Portugal, Tel: +3519148893 41; E-mail: rosacoelhoabrantes@hotmail.com

\begin{abstract}
Introduction: Autoimmune hepatitis $(\mathrm{AIH})$ and systemic lupus erythematosus (SLE) are both auto-immune disorders. Differential diagnosis between HAI and SLE can be a challenging task as there are some clinical and laboratorial findings co-existing between both pathologies.

Case description: The authors report a 37-years-old female patient who presented with oral ulcers, asthenia, anorexia, polyartralghia and arthritis with 3 months of evolution. Laboratory work-up revealed haemolytic anaemia, increased cytolic liver enzymes, positive anti-nuclear antibody, anti-neutrophil cytoplasmic antibody-MPO and anti-ds DNA test. Liver biopsy revealed a marked expansion of port tract by abundant fibrosis, mononuclear infiltrate and interface hepatitis. According to American College of Rheumatology criteria the patient has the diagnosis of SLE, fulfilling 4 of 11 criteria (oral ulcers, arthritis, haemolytic anaemia, and positivity of antinuclear and anti-ds DNA antibodies). The diagnosis of AIH was confirmed after calculating the international score for AIH Group (16 points).
\end{abstract}

Conclusions: The authors describe a case where the diagnosis of SLE is made in a patient with HAI. The coexistence of both pathologies is rare and differential diagnosis is a clinical challenge. This case highlight that in the presence of abnormal liver tests in a patient with SLE liver biopsy should be performed in order to exclude AIH as both diseases can co-exist.

Key-Words: Autoimmune Hepatitis; Systemic Lupus Erythematosus; Overlap Syndrome

\section{Introduction}

Auto-immune hepatitis (AIH) first described by Waldenstrom in 1950 [1] is characterized by continuing hepatocellular inflammation and necrosis, presence of circulating autoantibodies: antinuclear (ANA), smooth muscle (ASMA) and anti-liver-kidney microsome type 1 (anti-LKM1) antibodies associated with high serum of gamma globulin concentration [2]. The term lupoid hepatitis was introduced in 1955, when Joske et al reported the association between lupus erythematosus cell phenomenon in active chronic viral hepatitis [3].

However, nowadays, as we know, there is not a direct link between the two pathologies.
Systemic lupus Erythematosus (SLE) is a chronic inflammatory disease that affects multiple organs and systems such as skin, joints, kidneys, cardiovascular and central nervous systems. Twenty five to fifty percent of the patients with the diagnosis of SLE develop abnormal liver test during the course of the disease, being the main causes: hepatotoxic drugs, viral hepatitis and fatty liver disease [4]. In fact, the hepatic involvement by SLE, namely lupus hepatitis, is considered a rare condition [5] and the abnormalities of liver tests are not diagnostic criteria of SLE. After exclusion the other aetiologies of liver disease the definite differentiation between AIH and SLE with hepatic involvement is usually complex. As matter of fact, the difference between the hepatic involvement in SLE and AIH is not clearly defined yet. Similarities in the clinical and biochemical features of both diseases make the definitive diagnosis only possible after performing liver biopsy [6].

Nonetheless, antibodies against ribossomal P protein (anti-P) were recently considered a possible useful marker to differentiate lupus hepatitis from AIH. These antibodies were found in $44 \%$ patients with SLE and in patients in whom both disease co-exist this antibody was present in $20 \%$ of the patients [7]. However, in patients with AIH only it seems to be absent [7].

We report a female patient that fulfilled the criteria for SLE (defined by the American College of Rheumatology) and for AIH (considered by the International Group of AIH). The liver biopsy was performed and was compatible with the last diagnosis. This case highlights the complexity of the diagnosis of both disorders in the same patient that is considered a rare situation only few case reports have been published so far [8].

\section{Case report}

A 37-years-old female patient was referred to our hospital with complains of asthenia and anorexia, polyartralghia and arthritis in 3 joints, with 3 months of evolution. She mentioned past sulfonamide antibiotics allergy. She did not take any medication and denied personal history of auto-immune diseases; however, her mother had the diagnosis of SLE.

Clinical examination showed inflammatory signs in metocarphalangeal joints and both knees, and she was obese 
with a body mass index of $30.5 \mathrm{~kg} / \mathrm{m}^{2}$. The laboratory work-up revealed anaemia $(\mathrm{Hb}: 8.7 \mathrm{~g} / \mathrm{dL})$ with normal leukocyte $(6,79$ $\left.\mathrm{X} 10^{9} / \mathrm{L}\right)$ and platelet $(201 \mathrm{X} 10 / \mathrm{L})$ counts. A peripheral blood analysis showed schistocytes and the direct coombs test was positive with haptoglobin, total bilirubin and desidrogenase serum levels within the normal range. Biochemical liver workup revealed elevated serum alanine aminotransferase (ALT) 169U/L (N: 10-37), aspartate aminotransferase (AST) $143 \mathrm{U} / \mathrm{L}$ (N: 10-37), alkaline phosphatase 119U/L (30-120) and gammaglutamyl transpeptidase 227U/L (N: 10-49). Total bilirubin, albumin, total proteins, prothrombine time were normal. Ultrasound examination was unremarkable without biliary tract dilation Serology for hepatotrophic virus was performed, including: hepatitis A (IgM negative; IgG positive), B (HBsAg and $\mathrm{HBcAb}$ were negative with an HBsAb positive) and $\mathrm{C}$ (negative HCV antibodies) virus, Epstein-Barr (IgG positive; IgM negative), Herpes Simplex virus 1 and 2 (IgM negative; IgG positive, for both) and Cytomegalovirus (IgM negative; IgG positive). Metabolic hepatic diseases, hemochromatosis and Wilson disease, were ruled out with values of ceruloplasmin, $24 \mathrm{~h}$ urine cooper levels, iron and ferritin between the normal range. The immunological panel were marked of hypergammaglobulinaemia G [3650 mg/dL (N: 650 - 1500]. Serological tests were positive for serum positive anti-nuclear antibody(ANA)1/1000, antineutrophil cytoplasmic antibody-MPO: $72 \mathrm{U} / \mathrm{mL}(\mathrm{N}<20)$, anti-ds DNA>800U/L. Tests for ASMA, anti-LKM1, antiphospholip and anti-ribosomal $\mathrm{P}$ antibodies were negative.

Percutaneous liver biopsy was performed and revealed liver parenchyma with expansion of portal areas by marked fibrosis and presence of thick connective septa. Lesions of piecemeal necrosis (interface hepatitis) were also observed (figure 1).Using the international score for AIH our patient scored a total of 16 points, what is compatible with a definitive diagnosis of AIH in a pre-treatment picture.

Considering the American College of Rheumatology classification criteria for SLE the diagnosis of this auto-immune disease could be established as 4 of the 11 criteria were fulfil namely: presence of ANA, Anti-ds DNA, polyarthritis, oral ulcers and haemolytic anaemia.

Treatment with prednisolone $1 \mathrm{mg} / \mathrm{Kg} /$ day (60mg/day) and hidroxocloroquin $200 \mathrm{mg}$ per day were started. Clinical symptoms (asthenia, anorexia and polyarthritis) improved. The transaminases levels decreased in the first week after steroid treatment. The dose of steroids was tapered until $5 \mathrm{mg}$ per day and azathioprine was started. One year later, the patient was asymptomatic with normal liver tests.

\section{Discussion}

The diagnosis of AIH is based in a cluster of immunological results and blood analysis with an excellent response to immunosuppressive therapy. The simplified score to diagnosis AIH, which take into consideration only four items, was recognized and is used in every day clinical practise [10]. Nonetheless, for patients with atypical features the diagnostic scoring system for atypical autoimmune hepatitis in adults, established by the international Group, is the one that should be used [10].

The SLE is a chronic and inflammatory disease and its diagnosis is based in 11 criteria recognized by the American Society of Rheumatology. In order to make a definitive diagnosis of SLE 4 of these 11 criteria must be present [11].

However, even using these scoring systems the differential diagnosis between SLE and AIH is, in the majority of the cases, a changeling and difficult task. However, the definitive diagnosis is essential, as the complications of the two diseases are different. In AIH, the hepatic insufficiency is the terminal state, and in SLE the renal insufficiency is the main concern [8].

The authors describe a case of a female patient, with family history of SLE who describe being allergic to sulphonamides. She presented with recurrent oral ulcers, polyarthritis, haemolytic anaemia and positive immunological markers (ANA and Antids $D N A>800$ ). These 4 criteria allowed making the definitive diagnosis of SLE. Although not considered as criteria for SLE diagnosis the past medical history of allergy to sulphonamides was another positive finding for SLE diagnosis. In fact, the exposure to sulphonamide antibiotics is associated with an increased risk of exacerbation of SLE and described several times, incorrectly, as an "allergic" phenomenon.

One the other hand, and as our patient had abnormal cytolic enzyme pattern (3 times upper the normal lower limit), liver
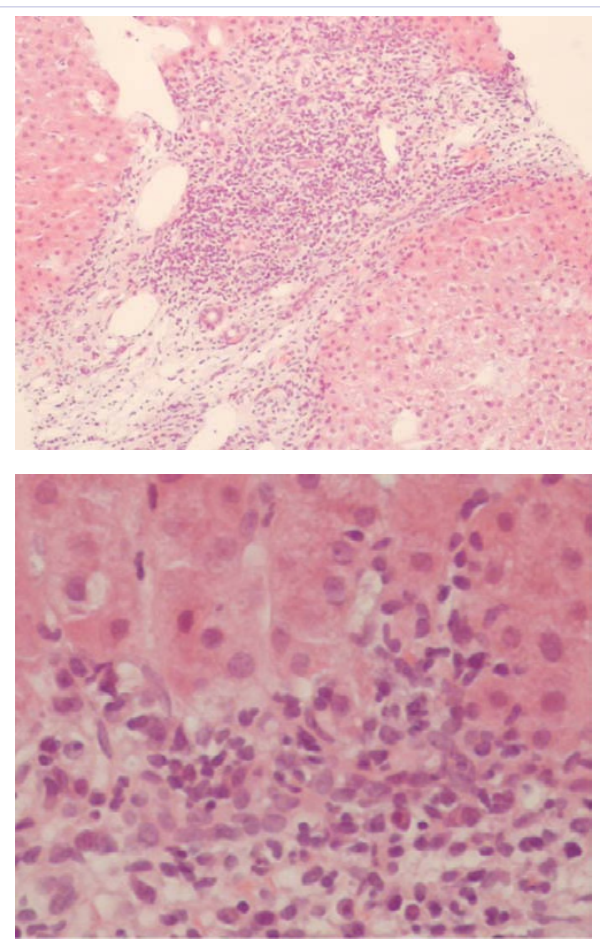

Figure 1: Liver biopsy showing liver parenchyma with expansion of portal areas by marked fibrosis and presence of thick connective septa (HE 100X). Lesions of piecemeal necrosis (interface hepatitis) HE 400X (B). 
biopsy was performed and revealed aport tract infiltration by mononuclear cells and interface hepatitis lesions, typical from AIH. In fact, the patient fulfilled 16 points in the international score for AIH corresponding to a definite diagnosis of $\mathrm{AIH}$. Therefore, the diagnosis of 2 concomitant auto-immune disorders was performed.

However, while SLE and AIH are rarely diagnosed as concomitant diseases in one patient, hepatic involvement in patients with SLE is sometimes observed during the course of disease. In these patients, AIH should be considered in the differential diagnosis of any SLE patient with elevated liver enzymes. Thus, liver histopathology represents the key feature and should become mandatory in such patients [7]. The histological findings that support an AIH are the presence interface hepatitis, rosetting of hepatocytes, emperipolesis and - consecutive to inflammation - fibrosis. The presence of lobar hepatitis or changes attributable either to drug toxicity or nonspecific liver involvement as e.g., fatty degeneration or hydropic hepatocytes are features more compatible with SLE [5, 7].

Recently, anti-ribosomal P antibody was considered an useful marker to differentiate SLE associated with hepatitis from AIH. The results from different studies are conflicting however it seems that the presence of anti-P antibodies is associated with multiple organ involvement: liver, kidneys, and central nervous system in patients with SLE [11]. Ohira et al reported that anti-P antibody was positive in $44 \%$ of the patients with SLE but negative in all tested patients with AIH [7]. In our patient, antiribosomal $\mathrm{P}$ antibody test was negative. This fact supports that liver abnormalities were more suggestive of an AIH and not due to liver involvement of SLE. Our patient was treated with steroid and azathioprine with normalization of laboratory findings and improvement of clinical symptoms. In fact, Efe et al recently described that patients with both auto-immune diseases showed a favourable response to therapy that included ursodeoxycholic acid, prednisolone, azathioprine or combinations of these [6].

In conclusion, we describe a case in which the AIH diagnosis was made in a patient who also fulfil the criteria for SLE. The corticoid response was excellent with disappearance of symptoms and normalization of liver test. This case highlight that in the presence of abnormal liver tests in a patient with SLE liver biopsy should be performed in order to exclude $\mathrm{AIH}$, as both diseases can co-exist.

\section{References}

1. Waldenstrom J. The diagnostic importance of ACTH. Acta Endocrinol (Copenh). 1950;5:235-242.

2. Krawitt EL. Autoimmune Hepatitis. N Engl J Med. 2006;354:54.

3. Joske RA, King WE. The L.E.-cell phenomenon in active chronic viral hepatitis. Lancet. 1955;269(6888):477-480.

4. Hallegua DS, Wallace DJ. Gastrointestinal manifestations of systemic lupus erythematosus. Curr Opin Rheumatol. 2000;12(5):379-385.

5. Beisel C, Weiler-Normann C, Teufel A, W Lohse A. An overlap syndrome involving autoimmune hepatitis and systemic lupus erythematosus in childhood. World J Gastroenterol. 2007;13(19):2764-2767.

6. Efe C, Purnak T, Ozaslan E, Ozbalkan Z, Karaaslan Y, Altiparmak E, et al. Autoimmune liver disease in patients with systemic lupus erythematosus: a retrospective analysis of 147 cases. Scand J Gastroenterol. 2011;46(6):732-737. doi: 10.3109/00365521.2011.558114.

7. Ohira H, Takiguchi J, Rai T, Abe K, Yokokawa J, Sato Y, et al. High frequency of anti-ribosomal $P$ antibody in patients with systemic lupus erythematosus associated hepatitis. Hepatol Res. 2004;28(3):137139.

8. Beisel C, Weiler-Normann C, Teufel A, Lohse AW. Association of autoimmune hepatitis and systemic lupus erythematodes: case series and review of the literature. World J Gastroenterol. 2014;20(35):12662-2667.

9. Hennes EM, Zeniya M, Czaja AJ, Parés A, Dalekos GN, Krawitt EL, et al. International Autoimmune Hepatitis Group. Simplified criteria for the diagnosis of autoimmune hepatitis. Hepatology. 2008;48(1):169-176.

10. Hahn BH, McMahon MA, Wilkinson A, Dean WallaceWD, Daikh DI, FitzGerald JD, et al. American College of Rheumatology Guidelines for Screening, Case Definition, Treatment and Management of Lupus Nephritis. Arthritis Care Res. 2012;64(6):797-808.

11. Carmona-Fernandes D, Santos MJ, Canhão H, Fonseca JE. Anti-ribosomal P protein IgG autoantibodies in patients with systemic lupus erythematosus: diagnostic performance and clinical profile. BMC Med. 2013;11:98. DOI: $10.1186 / 1741-7015-11-98$ 